\title{
Relationship between the Musculoskeletal System $\&$ High School Sports Injuries
}

\author{
Davie Rhaja A. Mariano \\ Newburgh Free Academy, daviemarianonetsci@gmail.com
}

\begin{abstract}
With participation in high school sports rising, the number of injuries sustained by high school athletes have also increased. This study sought to investigate the attributes of sports injuries and the common injury sites for male and female athletes to determine if certain muscles should be strengthened to prevent injuries in high school athletes. This project utilizes AnNA (anatomical network analysis) to show the relationships between the musculoskeletal system and high school sports injuries. An injury was defined as trauma to the musculoskeletal system. The study focused on the synovial joints of the human body and did not include head injuries. To do this we collected information from three data sets to develop three anatomical networks. The results showed that the articulation sites with the highest degree of muscle and bone account for the highest incidences of sports injury, most common injury site in female athletes was the ankle, but the most common injury site varied in male athletes. The results also showed that the anatomical differences between men and women account for the different injury sites. We hypothesize that strengthening the gastrocnemius muscle would help prevent injuries to knees and ankles, but further studies would need to be conducted.
\end{abstract}

Index Terms - anatomical network analysis, musculoskeletal, articulation, sports injury, synovial joints.

\section{Introduction}

I am a junior at Newburgh Free Academy and participating in the NetSci High program. NetSci High is a high school independent research program that uses network science to solve and analyze complex problems. In the summer of 2014, twenty-four students from four different high schools arrived at Boston to learn about network science. After learning about this "new" field of science, I told myself that I will incorporate networks in something that I love, sports and muscles. Many of my friends and family of mine have participated in sports and have been put on the sidelines due to injuries. Therefore, this was a topic that I wanted to explore and dedicate six months of research to.

\section{Background}

This study sought to investigate the relationships between high school students' sports injuries and the musculoskeletal system. To do this, we used the Anatomical network analysis (AnNA). We came across an article entitled Anatomical networks reveal the musculoskeletal modularity of the human head (Esteve-Altava, Diogo, Smith, Boughner \& Rasskin-Gutmanm, 2015) which utilized AnNA to create a more comprehensive understanding of the evolution of the human head. This study, our own personal interest in high school sports injuries, and the data from the RIO Injury Surveillance study (Comstock, Currie, \& Pierpoint, 2014) led us to conduct our own network research on the sports injury sites of high school athletes. This study defined an injury as harm done to the musculoskeletal system during a physical activity (Martini \& Nath, 2009). This study also focused on synovial joints, which have a wider 
range of movement than other joints. These joints, or articulations, are the most common areas of injuries, and include the following articulations: hip, ankle, knee, elbow, wrist, and shoulder. Head injuries were not included in this study. Questions asked during this study are as followed: Because movement is an important function to maintain homeostasis within the human body, where would the critical nodes for movement be located on a network of the human musculoskeletal system? Which articulation was more prone to injuries? Do male and female students who participate in similar sports incur the same sports injury? Which muscles could be used to strengthen the sports injury sites of high school students based on their highly connective articulation counterparts?

\section{Process}

This study collected data from: Fundamentals of Anatomy and Physiology textbook (Martini \& Nath, 2009) for information on articulations, www.SportInjuryClinic.net (2014) for information on the major muscles that attach the major articulation in the human body and the National High School SportsRelated Injury Surveillance Study 2013-2014 School Year (Comstock et. al, 2014) for statistical data on the common sporting injuries for male and female high school athletes. We used the data we collected and constructed the following networks: Musculoskeletal System, High School Athlete Injuries by Sport and Gender, and Network of Common High School Sports Injuries and Major Muscles. The Musculoskeletal System Network shows connections between the ligaments, bones and muscles found in the human body. The High School Athlete Injuries by Sport and Gender network compared number of injuries to each articulation for men and women by sport. With this we were able to explain why certain injuries occurred in females rather than males and vice versa. Finally, the Network of Common High School Sports Injuries and Major Muscles used the RIO Injury Surveillance data (Comstock, etc. al., 2014) and major muscle within those injury sites to show that muscles that are linked to highly connected articulations, should be strengthen in order to prevent injuries.

\section{Results}

Network of Common High School Sports Injuries and Major Muscles
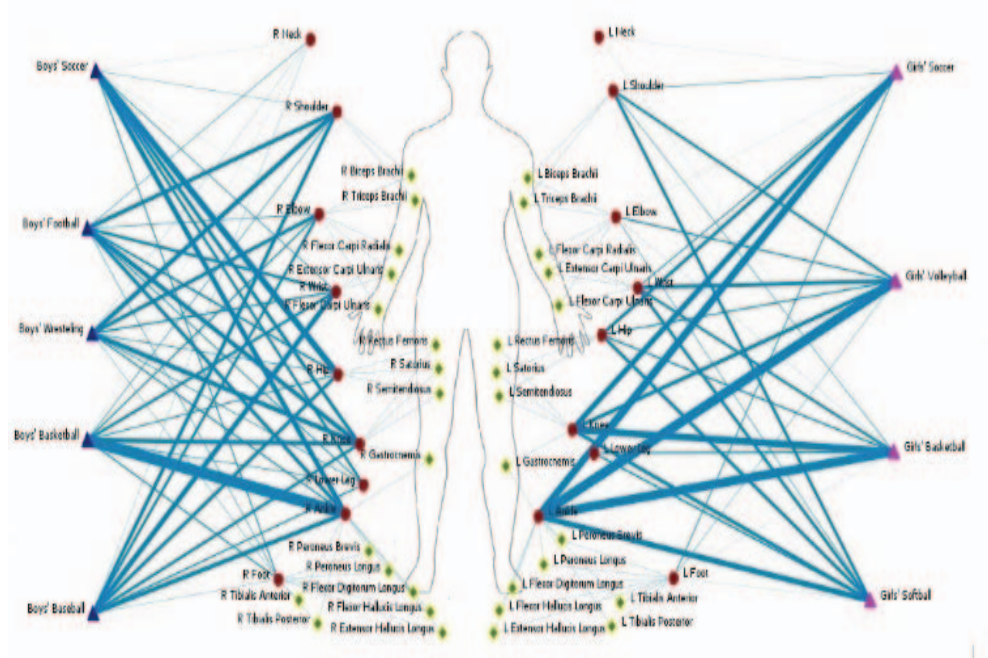

Figure 1. This network uses the RIO Injury Surveillance data (Comstock, etc. al., 2014) and major muscle with in those injury sites to show that muscles that are linked to highly connected articulations, should be strengthen in order to prevent injuries.

The Musculoskeletal System Network showed that the ankle had the highest degree with 12 and hip had the lowest with seven. This was odd because the hip is usually a stable joint and the ankle is usually more prone to injuries (Martini \& Nath, 2009). With that said, we 
realized that there must be more factors that contribute to it being more stable or more injury prone. The High School Athlete Injuries by Sport and Gender network showed that for female athletes, the ankle was the most injury prone articulation across all sports and for male athletes the most injury prone articulation varied by sport. We expected the articulation with the highest degree to vary by sport for both men and women. This could be caused by the anatomical difference between men and women. For example, females may be more susceptible to ankle injuries than males due to the difference in the alignment of their femurs. The Network of Common High School Sports Injuries and Major Muscles revealed that the gastrocnemius was the muscle with the highest eigenvector centrality. This suggests that it is the muscle that should be strengthened in order to prevent a large number of injuries. This was expected because the ankle and knee were hot spots for injuries and this was the only major muscle connecting the two articulations.

\section{Future Work}

We will create a preventative intervention plan that includes exercises to strengthen muscles connected to articulations with highest incidence of injuries, and conduct a study to determine the effectiveness of this course of action. 